\section{A Novel Device for the Clinical Assessment of Intranasal Trigeminal Sensitivity}

\begin{abstract}
Objective: Despite the significance of trigeminal pathology, practical clinical tests that accurately evaluate intranasal trigeminal function are scarce. The aim of the present study is to introduce a practical procedure for the assessment of intranasal trigeminal sensitivity.

Methods: We developed a device to stimulate the nasal mucosa using carbon dioxide, which is self-administered intranasally by holding down a timed button until the required sensory response has been triggered. The trigeminal sensitivity is derived from the measured administration time in conjunction with the concentration of carbon dioxide administered. Sixty-three healthy participants were used to validate the device, after which the new device was compared with a standard lateralization task in an additional 16 participants. In 20 participants, the experiment was repeated to verify test-retest reliability.

Results: Statistical analysis showed significant consistency in administration-duration in healthy individuals, including those in the test-retest group. Those participants with higher scores in the lateralization task were found to show higher intranasal sensitivity measured by the new device.

Conclusion: Herein, we present the design and validation of a novel device for the practical assessment of intranasal trigeminal sensitivity. In this study, we demonstrate the efficacy and reliability of this device.
\end{abstract}

\title{
Keywords
}

carbon dioxide, clinical, irritation, nasal, test, trigeminal

\section{Introduction}

Intranasal mucosal sensation is facilitated by the first 2 branches of the trigeminal nerve. Trigeminal receptors respond to a wide variety of substances, including capsaicin, menthol, gingerol, piperine, and carbon dioxide $\left(\mathrm{CO}_{2}\right)$, and these chemicals have been reported to elicit pain perception described as stinging, tingling, pungent, burning, or cooling. ${ }^{1}$ Most odorants activate trigeminal as well as olfactory receptors, and an interaction between the trigeminal and the olfactory system has been reported. ${ }^{2-4}$ In addition, the perception of nasal air flow is mediated by intranasal trigeminal sensation. ${ }^{5}$ Disruption or alteration of intranasal trigeminal sensory function can occur as a result of surgery (septoplasty, sinus surgery), intranasal pathology (inflammation, allergy, tumors), or therapeutic interventions (radioor chemotherapy).

A known stimulus specific to trigeminal receptors is $\mathrm{CO}_{2}$, which has been used to assess the antinociceptive effect of various analgesic drugs. ${ }^{6}$ However, current clinical assays that assess intranasal trigeminal function are unreliable or time consuming, indicating a need for a practical and effective test that can be integrated easily into standard outpatient care. The aim of the present study was to develop and validate an easy-to-use procedure for the evaluation of intranasal trigeminal sensitivity that enables the clinician to efficiently measure the psychophysical response to a trigeminal stimulus.

\section{Materials and Methods}

The study was carried out in the Department of Otorhinolaryngology at the Medical University of Vienna.

\footnotetext{
'Department of Otorhinolaryngology, Medical University of Vienna, Wien, Austria

${ }^{2}$ Department of Pharmacology, University of Erlangen-Nuremberg, Erlangen, Germany

Corresponding Author:

Christian A. Mueller, MD, Department of Otorhinolaryngology, Medical University of Vienna, Waehringer Guertel 18-20, Wien, 1090, Austria.

Email: christian.a.mueller@meduniwien.ac.at
} 
The study was conducted according to the guidelines of the Declaration of Helsinki on biomedical research involving human subjects and approved by the ethics committee of the Medical University of Vienna.

\section{Participants}

The investigation included 79 participants (40 male and 39 female, ages 18-81 years, with a mean age/standard error of the mean [SEM] of 31.1/1.7 years). The participants were sober and refrained from smoking for at least 1 hour prior to testing.

\section{Evaluation of Olfactory Function}

A standardized questionnaire was used to identify conditions that can cause olfactory dysfunction. Subsequently, physical examination of the nose was performed. Olfactory function was assessed with an odor identification test using 16 common odorants. ${ }^{7-9}$ Participants with severe olfactory dysfunction, septal deviation, or sinu-nasal disease were excluded from this group. Fifty-three of the 79 healthy participants were nonsmokers.

\section{Evaluation of Trigeminal Sensitivity}

Evaluation of intranasal trigeminal sensitivity was performed in 63 participants (34 male and 29 female, ages 18-78 years, with a mean age/SEM of $30.2 / 1.8$ years) with a novel device (trigeminometer), described below. The participants were asked to insert a small Teflon tube approximately $1 \mathrm{~cm}$ into 1 nostril (beyond the nasal valve as the posterior margin of the nasal vestibule) and breathe through the mouth. The following instructions were given: "Please breathe through your mouth and press the button just until you feel a clearly perceivable stimulus of medium strength that should not hurt much. That is a strength of approximately 5 on a scale from $0 /$ no pain to 10 /very painful." While the button was pressed, a valve switch was activated that guided the air flow (a mixture of $\mathrm{CO}_{2}$ and regular air) into the participant's nostril. The stimulus duration was recorded by a stopwatch connected to the button. To prevent a carry-over effect between nostrils, a minimum interval of 30 seconds was maintained prior to switching nostrils, allowing a minimum resting time for each nostril of 1 minute. Each nostril was tested using 3 different concentrations of $\mathrm{CO}_{2}(35 \%, 45 \%, 55 \%$; air flow $1 \mathrm{~L} / \mathrm{min}$ ), starting with 2 stimuli of the lowest concentration, resulting in a total of 6 stimuli for each side of the nose. The procedure was repeated with a 10-minute interval, to a total of 4 test measurements (measurements 1-4). No adverse effects were reported by any of the participants.

\section{Test-Retest Reliability Assessment}

To evaluate reliability of the device in the setting of repeated testing, the experiment was repeated at a later date in a subset of 20 healthy participants (range of retest, 1-56 days; mean/SEM, 20.3/4.7 days). The test results were matched and compared to the original scores and analyzed as detailed below.

\section{Comparison With a Standard Odor Lateralization Task}

Sixteen participants with no major nasal pathologies (10 female, 6 male; ages 21-81 years with a mean/SEM of $34.3 / 4.4$ years) were subjected to a pilot experiment in order to correlate trigeminal sensitivity using the novel device with an odor lateralization task. ${ }^{10}$ In a first session, they had to press 2 squeeze-bottles at the same time by a hand-held squeezing device delivering either odor or no odor to the right or left nostril in a randomized sequence. Participants had to name the side of stimulation in a forced-choice procedure. Two substances (pure odorant phenyl-ethanol [PEA], a rose-like odor; and a mixed trigeminal-olfactory substance, peppermint oil) were presented 20 times with a maximum score of 20 ( 1 point for each correct answer), respectively. In a second session, intranasal trigeminal sensitivity was assessed by the novel device as described above.

\section{Evaluation of Olfactory Function}

Olfactory function was assessed using odor-dispensing pens ("Sniffin' Sticks," Burghart Medical Technology, Wedel, Germany), filled with liquid odorants. The participants were instructed to sniff 16 different pens and match the smell with the correct descriptor from a list of 4 in a forced-choice manner. Each correct answer yielded 1 point, resulting in a maximum score of $16 .^{8}$

\section{Evaluation of Trigeminal Sensation}

The trigeminometer (Figure 1) consists of 2 flowmeters (Uniflux, V2 Fine Valve; Influx Measurements Ltd, Hampshire, UK) measuring $\mathrm{CO}_{2}$ (range, 0.3-2.8 L/min) and air (range, $0.2-2.0 \mathrm{~L} / \mathrm{min}$ ), respectively, a stopwatch (Digital Timer; Tele-Steuergeraete $\mathrm{GmbH}$, Hohenschaeftlarn, Germany), a valve switch (2/2-way small solenoid valve, type 6013; Buerkert GmbH, Vienna, Austria), pressurized cylinders for air and $\mathrm{CO}_{2}$ (Messer Medical Austria $\mathrm{GmbH}$, Gumpoldskirchen, Austria) fitted with pressure control valves (GCE-Rhoena Autogengeraete GmbH, Fulda, Germany), and connecting Teflon tubes (inner diameter, 4 $\mathrm{mm}$; outer diameter, $6 \mathrm{~mm}$ ). 


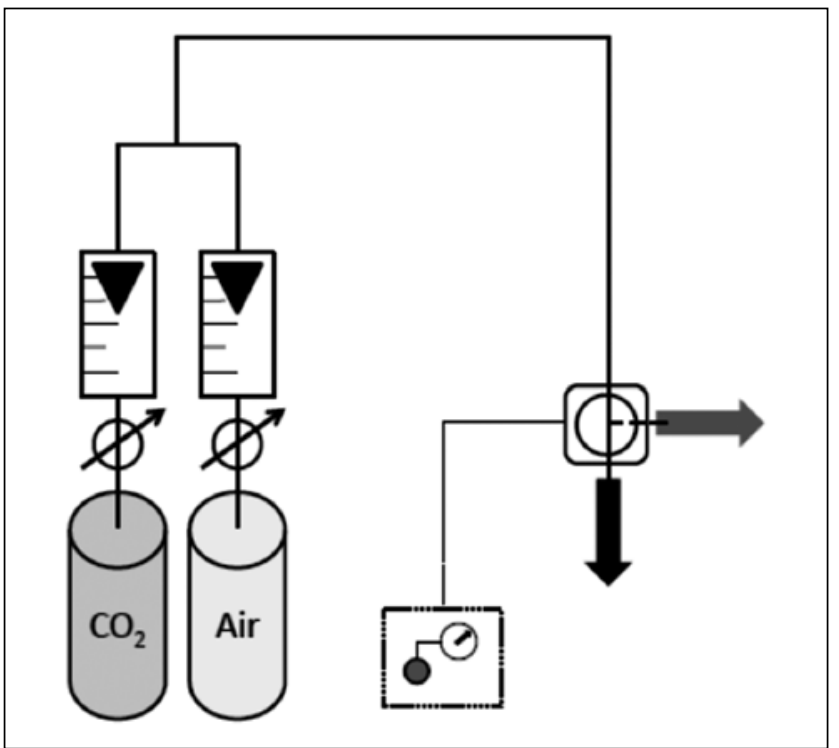

Figure I. Schematic drawing of the trigeminometer. Pressing the operation button activates the flow of mixed gas (air and carbon dioxide) into the participant's nostril (black arrow), while simultaneously starting a timer. When the button is released, the flow of gas is diverted to the outlet (gray arrow) and the timer is automatically stopped, recording the stimulation time.

The stopwatch was connected to the valve switch, enabling accurate timing of administration of the gas mixture into the nostril. The duration was recorded in seconds with an accuracy of one hundredth of a second. All interested readers needing support in building or purchasing the device are invited to contact the corresponding author. Detailed specifications of the technical parts including the wiring diagram of the control unit will be provided. The price of the trigeminometer (consisting of 2 flowmeters, the stopwatch, and valve switch with connecting tubes) is approximately $\$ 900$ USD.

\section{Statistical Analysis}

SigmaPlot version 11.0 (Systat Software Inc, San Jose, California, USA) was used for analyses and graphical presentation. Data were tested for normality using Shapiro-Wilk test. Analysis of variance (ANOVA) was performed to compare duration of stimulation using the 3 different concentrations of $\mathrm{CO}_{2}$, between individual measurements and nostrils. Analysis of variance was also used to determine differences between repeated measurements obtained from each nostril and to explore effects of sex and smoking habits. MannWhitney rank sum test was performed to test for significant differences between the nostrils. Wilcoxon signed-rank test for paired samples was used to compare the values of the 20 test-retest reliability participants tested on 2 different days. Spearman's and Pearson's statistics were used for correlation analysis. A $P$ value $<.05$ was considered significant.
Table I. Stimulus Durations. ${ }^{\text {a }}$

\begin{tabular}{|c|c|c|c|}
\hline & Low & Medium & High \\
\hline \multicolumn{4}{|c|}{ Measurement I } \\
\hline Right & $1.43 \pm 0.12^{b}$ & $0.84 \pm 0.06^{b}$ & $0.65 \pm 0.06^{b}$ \\
\hline Left & $1.36 \pm 0.12^{c}$ & $0.90 \pm 0.08^{c}$ & $0.58 \pm 0.04^{c}$ \\
\hline \multicolumn{4}{|c|}{ Measurement 2} \\
\hline Right & $1.28 \pm 0.09^{b}$ & $0.89 \pm 0.07^{b}$ & $0.68 \pm 0.09^{b}$ \\
\hline Left & $1.28 \pm 0.09^{c}$ & $0.88 \pm 0.06^{c}$ & $0.57 \pm 0.04^{c}$ \\
\hline \multicolumn{4}{|c|}{ Measurement 3} \\
\hline Right & $1.29 \pm 0.09^{b}$ & $0.88 \pm 0.07^{b}$ & $0.65 \pm 0.05^{b}$ \\
\hline Left & $1.25 \pm 0.08^{c}$ & $0.82 \pm 0.06^{c}$ & $0.59 \pm 0.04^{c}$ \\
\hline \multicolumn{4}{|c|}{ Measurement 4} \\
\hline Right & $1.38 \pm 0.10^{\mathrm{b}}$ & $0.88 \pm 0.07^{b}$ & $0.64 \pm 0.05^{b}$ \\
\hline Left & $1.34 \pm 0.08^{c}$ & $0.93 \pm 0.07^{c}$ & $0.63 \pm 0.05^{c}$ \\
\hline
\end{tabular}

${ }^{a}$ Duration (seconds) of intranasal stimulation using 3 different concentrations of carbon dioxide $\left(\mathrm{CO}_{2}\right)$ in healthy participants $(\mathrm{N}=63)$. Values indicate mean duration \pm standard error of the mean (SEM).

bStatistically significant difference between all $\mathrm{CO}_{2}(P<.00 \mathrm{I})$ comparing the 3 stimulus concentrations (right nostril).

'Statistically significant difference between all $\mathrm{CO}_{2}(P<.00 \mathrm{I})$ comparing the 3 stimulus concentrations (left nostril).

\section{Results}

\section{Evaluation of Olfactory Function}

Odor identification test scores ranged from 11 to 16 points with a mean of 14.1 points and a SEM of 0.1 points. One participant scored "hyposmic," as compared to normative data described elsewhere. ${ }^{11}$

\section{Evaluation of Intranasal Trigeminal Function}

Stimulus durations of all measurements on the first day ranged from 0.18 to 6.08 seconds for the lowest, from 0.21 to 4.46 seconds for the medium, and from 0.04 to $5.21 \mathrm{sec}-$ onds for the highest concentration of $\mathrm{CO}_{2}$. The mean latency values for all measurements are shown in Table 1 . Analysis of variance yielded significant differences in stimulus duration with all 3 concentrations of $\mathrm{CO}_{2}$ in each of the 4 measurements $(P<.001)$. A graphical representation is shown in Figure 2. Multivariate analysis did not show significant correlations between stimulus duration and the participant's age, left versus right side of stimulation, smoking habits, or sex.

\section{Assessment of Test-Retest Reliability}

Twenty participants were subjected to retesting after a varying amount of days, to assess test-retest reliability of our assay. Stimulus durations recorded on different days in 20 participants showed high correlation (Pearson $r_{480}=0.69$; $P<.001$ ), but there was no overall effect between both days as expected by the correlation analysis. A further post hoc 

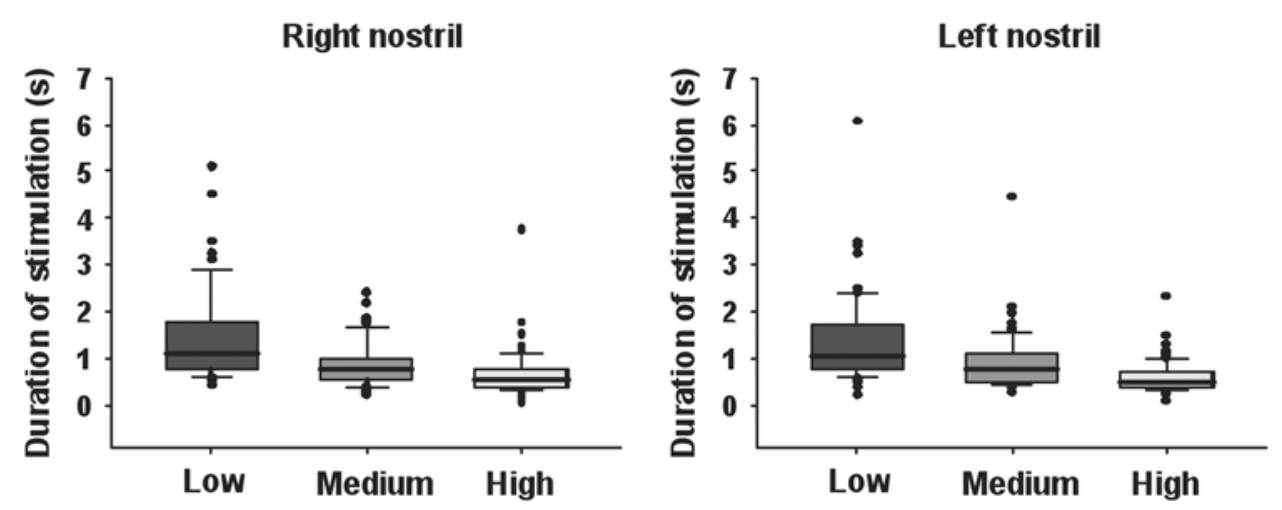

Figure 2. Stimulus durations. Duration (seconds) of intranasal stimulation (measurement $I, N=63$ ) with varying concentrations of carbon dioxide ( $\mathrm{CO}_{2}$; low, medium, high) displayed per nostril. Thick bars within the box plots represent the 50th percentiles (median), the upper and lower edges of the box plots indicate the 25th and 75th percentiles, respectively, and the whiskers show the 10th and 90th percentiles. Dots represent outliers. Significant differences in the duration of stimulation were found with all 3 concentrations on both sides of the nose $(P<.001)$.

analysis for inter-day differences yielded no significant differences for all measurements, except 1 (left side, medium concentration, measurement 3 , first day mean/SEM: 0.82/0.09 seconds; second day mean/SEM: 0.98/0.09 seconds, $P=.046$; not corrected for multiple comparisons).

\section{Correlation of the Odor Lateralization Task and the Trigeminometer}

The mean score/SEM of the lateralization task was 12.5/1.0 for PEA and 15.3/1.1 for peppermint oil. Statistical analysis showed a significant negative correlation between the results of the lateralization task using peppermint oil and the stimulus duration assessed with the highest $\left(r_{16}=\right.$ $-0.589, P=.016)$ and the medium concentration of $\mathrm{CO}_{2}\left(r_{16}=\right.$ $-0.526, P=.036)$ but not with the lowest concentration $\left(r_{16}=-0.276, P=.301\right)$. As expected, no correlations were observed for PEA and the $3 \mathrm{CO}_{2}$ conditions $\left(r_{16}=-0.03\right.$ to $-0.192, P=.476-.913)$.

\section{Discussion}

In the present study, we aimed to develop a new method for the clinical evaluation of intranasal trigeminal sensitivity. First, a device was developed to selectively stimulate trigeminal nerve fibers, which was achieved by the use of $\mathrm{CO}_{2}$ as the stimulant. ${ }^{12,13}$ Operation of our device is easy, and an examination takes only minutes in contrast to most established procedures for the assessment of intranasal trigeminal function. ${ }^{14}$ The strength of stimulation can be adjusted by changing the $\mathrm{CO}_{2}$ component of the gas mixture, allowing for a broad range of sensitivity assessment. Preliminary data led to the selection of 3 different concentrations, ranging from mild to strong stimulation without causing excessive discomfort. The apparatus is designed to be operated by the participant, avoiding the possibility of the examiner overstimulating the participant. In addition, the intention was to give the participants control of stimulation, so that the self-adjusted duration of application of $\mathrm{CO}_{2}$ into the nose could be measured.

The second part of the study involved validation of our device through 2 distinct participant groups: nontrained healthy participants in order to gain normative values of the novel device, and additional participants performing an odor lateralization task in comparison with the trigeminometer. We tested different concentrations of stimulant $\mathrm{CO}_{2}$ to evaluate the effective range of the device. Our results showed that all 3 concentrations of $\mathrm{CO}_{2}$ could be discriminated by the participants and that the measurements were reproducible when tested in repeated sessions.

Regarding the odor lateralization task, our results showed random lateralization with the pure odorant PEA. This is in accordance with the fact that pure odorants cannot be attributed to 1 side of the nose when presented simultaneously to both sides of the nose..$^{15}$ Accordingly, no correlations were visible between the lateralization task and stimulus durations assessed by the novel device. The second odorant with trigeminal input (peppermint oil) yielded lateralization above chance level. As expected, participants with better lateralization scores showed higher trigeminal sensitivity using the novel device. Therefore, we observed a negative correlation between stimulus duration after high/ medium $\mathrm{CO}_{2}$ application and the lateralization score of peppermint. Due to the higher data variability after stimulation with the lowest $\mathrm{CO}_{2}$ concentration (see Table 1 and Figure 2) and due to the limited number of participants in our pilot 
study, the correlation of lateralization scores and results with low $\mathrm{CO}_{2}$ was not significant. Nevertheless, the device provided reliable data as compared to a standard procedure for the assessment of trigeminal sensitivity. The rapid application time of only a few minutes compared to approximately 10 minutes of the lateralization task seems to be an advantage of the new method. Moreover, the concentration of the $\mathrm{CO}_{2}$ /air mixture can be adjusted easily in a broad range, thus leading to the advantage of conducting experiments in a shorter period of time.

Our novel modality has several additional advantages when compared to the current standard. Whereas established psychophysical procedures like the described nasal lateralization task or eye irritation tasks employ squeezebottles of several different concentrations ${ }^{16}$ or make use of $\mathrm{CO}_{2}$-induced changes in the respiratory pattern, ${ }^{17}$ our method induces little discomfort, is less laborious, has a shorter duration, and still maintains accuracy and specificity. Moreover, participant instruction is straightforward and simple, and the method of self-administration decreases examiner-derived bias while introducing a minimal amount of subjectivity.

The novel apparatus provided highly significant and stable differences in application for all 3 concentrations of $\mathrm{CO}_{2}$. Repeated measurements showed stable stimulus durations in the tested participants, whereas test-retest reliability evaluation did not show differences in any of the measurements except in 1 case. The inter- and intra-individual differences found in stimulus duration will allow for the comparison of anatomical and physiological variation in future studies. Differences in stimulus duration between the left and right nostrils in patients with a deviated septum might be due to anatomical asymmetry of the nasal cavity. When interpreting differences in sensitivity in the context of generalized intranasal pathology, the composition of the nasal mucosa (eg, in allergic and chronic atrophic rhinitis) might be an influencing factor. Specifically, patients' perception of impaired nasal breathing might be due to not only reduction in airflow but also an altered perception of nasal airflow. One possible reason is pathological turbulence in the nasal cavity leading to drying of the mucosa and formation of crusts. Therapy in these patients aims at cleaning and moisturizing the nasal mucosa. Because measurement of nasal airflow alone does not provide information about changes in mucosa composition during therapy, the novel device might help to quantify the effects of therapy in these patients, which means that trigeminal sensitivity might change without a change in nasal airflow in certain patients. Other possibilities for further investigation include correlating these results with measured parameters of nasal breathing (eg, using rhinomanometry) or anatomic measures like acoustic rhinometry. Moreover, differences in personality and state of anxiety might have an additional effect on trigeminal sensitivity. Besides comparing participant groups with different characteristics, examination of intra-individual changes in intranasal trigeminal sensitivity within a specific group (such as patients who have undergone surgical or noninvasive interventions [eg, analgesics, psychological treatment]) might be performed using our device. Our results warrant further investigation into the application of the new trigeminometer in pharmacological studies.

\section{Conclusion}

We introduced a practical device for the clinical assessment of intranasal trigeminal sensitivity that may provide new insights into the pathological mechanism of diseases involving the trigeminal nerve. Future investigation should yield normative values derived from a larger sample of participants and should focus on expanding the possible application of this novel approach.

\section{Authors' Note}

This study was approved by the local ethics committee (EK-Nr. 359/2006, 1142/2010).

\section{Declaration of Conflicting Interests}

The author(s) declared no potential conflicts of interest with respect to the research, authorship, and/or publication of this article.

\section{Funding}

The author(s) received no financial support for the research, authorship, and/or publication of this article.

\section{References}

1. Silver WL, Finger TE. The anatomical and electrophysiological basis of peripheral nasal trigeminal chemoreception. Ann N Y Acad Sci. 2009;1170:202-205.

2. Hummel T, Livermore A. Intranasal chemosensory function of the trigeminal nerve and aspects of its relation to olfaction. Int Arch Occup Environ Health. 2002;75:305-313.

3. Leopold DA, Hornung DE, Schwob JE. Congenital lack of olfactory ability. Ann Otol Rhinol Laryngol. 1992;101:229-236.

4. Frasnelli J, Hummel T. Interactions between the chemical senses: trigeminal function in patients with olfactory loss. Int J Psychophysiol. 2007;65:177-181.

5. Eccles R. Nasal airway resistance and nasal sensation of airflow. Rhinol Suppl. 1992;14:86-90.

6. Renner B, Clarke G, Grattan T, et al. Caffeine accelerates absorption and enhances the analgesic effect of acetaminophen. J Clin Pharmacol. 2007;47:715-726.

7. Kobal G, Hummel T, Sekinger B, Barz S, Roscher S, Wolf S. "Sniffin' sticks": screening of olfactory performance. Rhinology. 1996;34:222-226.

8. Hummel T, Sekinger B, Wolf SR, Pauli E, Kobal G. 'Sniffin' sticks': olfactory performance assessed by the combined testing of odor identification, odor discrimination and olfactory threshold. Chem Senses. 1997;22:39-52. 
9. Hummel T, Konnerth CG, Rosenheim K, Kobal G. Screening of olfactory function with a four-minute odor identification test: reliability, normative data, and investigations in patients with olfactory loss. Ann Otol Rhinol Laryngol. 2001;110: 976-981.

10. Hummel T, Futschik $T$, Frasnelli J, Hüttenbrink KB. Effects of olfactory function, age, and gender on trigeminally mediated sensations: a study based on the lateralization of chemosensory stimuli. Toxicol Lett. 2003;140-141: 273-280.

11. Hummel T, Kobal G, Gudziol H, Mackay-Sim A. Normative data for the "sniffin' sticks" including tests of odor identification, odor discrimination, and olfactory thresholds: an upgrade based on a group of more than 3,000 subjects. Eur Arch Otorhinolaryngol. 2007;264:237-243.
12. Kobal G. Pain-related electrical potentials of the human nasal mucosa elicited by chemical stimulation. Pain. 1985;22:151-163.

13. Hummel T, Kraetsch HG, Pauli E, Kobal G. Responses to nasal irritation obtained from the human nasal mucosa. Rhinology. 1998;36:168-172.

14. Hummel T. Assessment of intranasal trigeminal function. Int J Psychophysiol. 2000;36:147-155.

15. Kobal G, Van Toller S, Hummel T. Is there directional smelling? Experientia. 1989;45:130-132.

16. Cometto-Muniz JE, Cain WS. Relative sensitivity of the ocular trigeminal, nasal trigeminal and olfactory system to airborne chemicals. Chem Senses. 1995;20:191-198.

17. Shusterman DJ, Balmes JR. A comparison of two methods for determining nasal irritant sensitivity. Am J Rhinol. 1997;11:371-378. 